\title{
Presentación:
}

\section{Desarrollo Profesional basado en la escuela y en el Territorio}

\section{School-based and Territory-based Professional Development}

\author{
Cecilia Simón 1, * \\ Yolanda Muñoz ${ }^{2}$ \\ ${ }^{1}$ Universidad Autónoma de Madrid, España \\ ${ }^{2}$ Universidad de Alcalá, España
}

Los centros que se comprometen con un proceso de revisión y mejora con la finalidad de ser más inclusivos y que logran avanzar en este propósito de una forma sostenible, tienen en común, entre otras cosas, que se preocupan por el desarrollo profesional continuado de su equipo docente. Esta es una de las condiciones importantes para el avance de los centros. El desarrollo de las competencias que necesita un profesorado preocupado por articular con equidad la presencia, el aprendizaje y la participación de todo su alumnado (Ainscow et al., 2006) debe ser uno de los propósitos de los sistemas educativos, tanto en lo relacionado con la formación inicial como continua. Un profesional que, como se desprende del perfil del profesorado para la inclusión propuesto por la Agencia Europea para las necesidades educativas especiales y la inclusión educativa, en el proyecto que desarrollaron denominado "Formación del Profesorado para la Inclusión" (TE4I) debe (AEDNEEI, 2011): a) valorar la diversidad del alumnado (las diferencias en el aprendizaje deben ser vistas como como un recurso y un valor educativo), b) apoyar a todos los aprendices (el profesorado debe tener altas expectativas sobre el rendimiento de todo su alumnado), c) trabajar con otros (la colaboración y el trabajo en equipo son esenciales para la tarea que todos los profesores deben desarrollar), y d) cuidar el desarrollo profesional y personal continuo (la enseñanza es una actividad de aprendizaje constante y el profesorado debe asumir la responsabilidad de su formación permanente, a lo largo de su vida profesional).

Sin duda, el desarrollo de prácticas docentes con el propósito de "llegar a todo el alumnado", sin dejar a "nadie atrás" se constituye en uno de los requisitos para el logro del reto internacional que hemos asumido en el Objetivo no 4 de la Agenda 2030 para el Desarrollo Sostenible. Sin embargo, la distancia que nos queda por recorrer para alcanzar este Objetivo (UNESCO, 2020 a), nos insta a tener muy presentes las acciones necesarias para avanzar (Echeita et al., 2021). Con esta finalidad, entre otras cosas, como señala la UNESCO (2020b), los sistemas educativos deberían esforzarse por apoyar a las escuelas y a los docentes, contribuyendo al desarrollo de sus competencias para llegar a la diversidad del alumnado de su centro, y fomentando la colaboración entre los diferentes agentes relacionados con la educación y bienestar de sus alumnos y alumnas, tanto dentro de la escuela como fuera de esta.

*Contacto: cecilia.simon@uam.es 
A este respecto y centrándonos en el profesorado en ejercicio, hay importantes preguntas a las que una investigación rigurosa puede y debe ayudar a contestar: ¿qué estrategias pueden utilizar o poner en marcha los centros para lograr este propósito?, ¿cuáles resultan más eficaces?, ¿qué condiciones escolares las propician y qué barreras las obstaculizan?, ¿cómo se facilita su sostenibilidad y generalización en el propio centro y en otros? o ¿qué tipo de relaciones se pueden establecer con los distintos agentes y organizaciones del entorno para fomentar dicho desarrollo? Estas son las preguntas que han guiado los diferentes textos que se presentan. Su objetivo es contribuir a la reflexión con evidencias en torno a estas y otras preguntas cuyas respuestas puedan contribuir a la implementación de estrategias para dicho desarrollo profesional, sea desde la propia institución educativa o desde la construcción de alianzas con el territorio. Como podremos apreciar, desde una mirada amplia y sistémica de apoyo (Booth y Ainscow, 2015), la utilización de los recursos existentes en el propio centro, como es la colaboración entre docentes o entre estos y otros miembros del centro escolar como el alumnado, así como los existentes en el territorio, será estratégico a la hora de introducir mejoras sostenibles en las escuelas.

Los diferentes artículos que presentamos en la sección temática de este número de la Revista Latinoamericana de Educación Inclusiva vienen a dar respuesta a la aportación que tiene la investigación inclusiva para el desarrollo profesional docente y nos ofrecen una amplia perspectiva desde diferentes países. De este modo, a través de varios artículos que analizan y reflexionan sobre esta cuestión se da paso a experiencias prácticas que ahondan en diferentes estrategias que promueven el desarrollo profesional de los docentes en los centros educativos.

En el artículo "Inclusive inquiry: an innovative approach for promoting inclusion in schools", se encontrará una propuesta de revisión y mejora para la inclusión en los centros basada en la participación de los estudiantes en las escuelas como investigadores, que colaboran con su profesorado y compañeros en el diseño de lecciones inclusivas.

A continuación, en el artículo "Pensar el desarrollo profesional docente desde la investigación: rutas participativas e inclusivas" se reflexiona sobre cómo la investigación participativa e inclusiva, entendida desde una mirada alterativa de cómo el investigador se acerca a la inclusión educativa, contribuye al desarrollo profesional docente desde los resultados de tres proyectos de investigación orientados hacia la inclusión a través de la creación de redes de carácter comunitario con acciones socio-educativas concretas.

Las redes de carácter comunitario dejan paso a la "Formación permanente del profesorado para la inclusión educativa, mediada por narraciones fotográficas”, artículo en el que se analiza el papel que la práctica sistemática de narraciones fotográficas puede tener en el desarrollo profesional docente. Entendiendo esta como una estrategia fundamental para la formación del profesorado en los ámbitos de la participación y de las relaciones internas y externas del centro que fomentan la educación inclusiva. Llevar a cabo indagaciones visuales a través de relatos fotográficos ofrece una oportunidad única para explorar y comprender el contexto social y escolar de los centros educativos, y hacerlo juntos conlleva la promoción de relaciones interpersonales cercanas, desde el conocimiento mutuo y el respeto a las distintas realidades.

El artículo "Desarrollo profesional docente para la inclusión: investigación acción colaborativa a través de estudios de clase en escuelas chilenas" profundiza sobre lo que aportan los "estudios de clase" en el desarrollo profesional de los docentes a través de los resultados de un estudio llevado a cabo en Chile, destacando el papel fundamental que tiene para este 
desarrollo el que el profesorado pueda llevar a cabo procesos reflexivos sobre la acción educativa.

Por su parte, el trabajo titulado "Creación de una estructura colaborativa entre el alumnado, docentes e investigadores: impacto en la mejora docente" parte del enfoque que se propone en el primer artículo del monográfico, tratando de mostrar, precisamente, la eficacia de la creación de una estructura de diálogo entre estudiantes y profesorado con la finalidad de mejorar las prácticas docentes en el sentido de articular con equidad la presencia, el aprendizaje y la participación de todo el alumnado sin dejar a nadie atrás.

Para el análisis de la importancia de los procesos reflexivos del profesorado contamos con el artículo "La reflexión colectiva entre profesoras en formación inicial y continua como espacio de construcción de una pedagogía inclusiva”. En este texto se muestra la reflexión colectiva sobre la práctica entre profesorado en formación inicial y continua como estrategia para la construcción de una pedagogía inclusiva, a partir de los principios del diseño universal para el aprendizaje.

En el siguiente artículo "Caminando hacia la inclusión a través de la investigación-acción participativa en una comunidad educativa" se presenta la experiencia concreta de un centro educativo en España que ha puesto en marcha una investigación acción participativa para el desarrollo de una educación inclusiva, siendo los principales agentes de cambio el profesorado del centro educativo. Para ello, a través de distintas fases de este tipo de investigación, se presenta un proceso de cambio de un centro educativo desde una perspectiva inclusiva, que puede arrojar luz sobre cómo otros pueden iniciar esos procesos y algunos de los resultados más relevantes.

A continuación, el artículo "Dimensiones clave para el desarrollo escolar inclusivo: reflexiones desde una experiencia de asesoramiento colaborativo" contextualizado en centros educativos de Chile, analiza cinco dimensiones clave en los procesos de cambio educativo hacia modelos más inclusivo que incluyen las culturas colaborativas, la investigación-acción participativa que ha sido objetivo de los dos artículos previos, el liderazgo inclusivo, la visión de cambio integral y finalmente el asesoramiento colaborativo. Esta última dimensión se establece como una de las principales para superar algunas de las barreras para la educación inclusiva en cuanto a cómo se concibe el currículo y la diversidad en los centros educativos.

El papel que pueden desempeñar ciertos servicios externos, tradicionalmente entendidos como recursos exclusivos para atender a alumnado de ciertos colectivos tradicionalmente vulnerables como pueden ser los estudiantes con discapacidad, en el desarrollo profesional docente hacia una educación más inclusiva es el objeto del siguiente artículo. Este artículo contextualizado en México que se titula "Sistematización de una experiencia de inclusión educativa en el nivel de Educación Secundaria, como estrategia para la formación docente y el desarrollo de proyectos colectivos", nos presenta los resultados de la colaboración entre el Servicio de Apoyo a la Educación Regular y un centro educativo de Educación Secundaria.

Cuando hablamos de desarrollo profesional docente, no debemos olvidar el papel que puede tener la puesta en marcha de metodologías más activas e inclusivas en el aula en el desarrollo del mismo. Así, en el siguiente artículo "Una experiencia de aprendizaje por proyectos cooperativos para la inclusión en un centro de Educación Primaria: visiones docentes y discentes" se analizan los resultados de la puesta en marcha de la metodología de 
aprendizaje cooperativo en dos grupos clase de un centro educativo de España, centrada en el desarrollo de una enseñanza más inclusiva.

Finalmente, un elemento esencial para el desarrollo profesional docente en el marco de una educación inclusiva, y que surge de manera transversal en varios de los artículos de este monográfico, es el liderazgo inclusivo. En ello entra de lleno el último artículo "Relaciones colaborativas entre docentes y directivos: un estudio de caso sobre el desarrollo profesional e inclusión educativa en pandemia", que además se contextualiza en el periodo de pandemia que estamos viviendo con las enormes barreras que ello ha supuesto en Chile. Las prácticas colaborativas entre docentes y entre docentes y directivos son el objeto de este artículo que nos adentra en la necesidad de fomentar prácticas colaborativas para el desarrollo de las escuelas, especialmente en contextos especialmente complejos o retadores.

Por otra parte, la sección de temática libre de la revista incluye dos interesantes investigaciones. El primer trabajo titulado "Efectos de la tutoría entre iguales como estrategia de educación inclusiva en el alumnado de educación infantil y primaria con Trastorno del Espectro Autista" (TEA), analiza los resultados de una rigurosa revisión de estudios sobre Tutoría entre Iguales, sus efectos y beneficios para el alumnado con TEA. Lo interesante de este trabajo es que muestra que, pese a ser ésta una metodología altamente recomendada para favorecer la inclusión, es una práctica muy poco estudiada con esta población, dando cuenta que la tutoría entre pares es efectiva para el desarrollo de las habilidades sociales, comunicativas y académicas de los estudiantes con TEA y como sus compañeros/as que ejercen el rol de tutores viven la experiencia de forma positiva, obteniendo también beneficios para su desarrollo personal.

Por último, el segundo artículo de tema libre con el que cerramos este número, aborda un tema de gran relevancia para la construcción de comunidades escolares inclusivas. Titulado "Diversidad sexual y de género en comunidades educativas de Arica, Chile: Fisura de la heteronorma desde la multicultura", este trabajo aporta a la comprensión de los significados sobre la diversidad sexual y de género que construyen comunidades educativas de educación básica y media de una región del norte Chile y que dan cuenta de las tensiones derivadas de una matriz heterosexual heredera de la hegemonía patriarcal en generaciones más adultas que toleran discursivamente las diversas identidades de género, pero las sancionan en la cotidianidad escolar, versus generaciones más jóvenes que las aceptan enfatizando en la urgencia de formación para la transformación social desde la inclusión y no discriminación.

\section{Referencias}

AEDNEEI. (2011). Formación del profesorado para la educación inclusiva en Europa. Retos y oportunidades. Agencia Europea para el Desarrollo de la Educación del Alumnado con Necesidades Educativas Especiales. http://www.europeanagency.org/sites/default/files/te4i-challenges-andopportunities_TE4I-Synthesis-ReportES.pdf

Ainscow, M., Booth, T. y Dyson, A. (2006). Improving Schools, Developing Inclusion. Routledge.

Booth, T. y Ainscow, M. (2015). Guía para la Educación Inclusiva. Desarrollando el aprendizaje y la participación en los centros escolares. OEI/FUHEM. 
Echeita, G., Simón, C., Muñoz, Y., Martín, E., Palomo, R. y Echeita, R. (2020). El papel de los Centros de Educación Especial en el proceso hacia sistemas educativos más inclusivos. Cuatro estudios de casos: Newham (UK), New Brunswick (Canadá), Italia y Portugal. Servicio de Publicaciones MEFP

UNESCO. (2020a). Inclusion and education: All means all. Global monitoring report. UNESCO.

UNESCO. (2020b). Towards inclusion in education: Status, trends and challenges. The UNESCO Salamanca Statement 25 years on. UNESCO.

\section{Breve CV de las autoras}

\section{Cecilia Simón Rueda}

Profesora Titular del Departamento Interfacultativo de Psicología Evolutiva y de la Educación de la Universidad Autónoma de Madrid (UAM). Es co- coordinadora del grupo de investigación "Equidad, diversidad y educación inclusiva" (EQUIDEI) de la UAM. Forma parte de Catalizadores para la Educación Inclusiva (Inclusion International) y ha colaborado con entidades como la OEI y la Agencia Europea para las Necesidades Educativas Especiales y la Inclusión Educativa. Sus intereses de investigación se relacionan con el desarrollo de una educación más inclusiva, así como con la relación escuela, familia y comunidad. Ha participado en múltiples proyectos de ámbito nacional e internacional sobre estos temas, siendo IP de alguno de ellos. Email: cecilia.simon@uam.es

ORCID ID: https://orcid.org/OOOO-0002-1795-9109

\section{Yolanda Muñoz Martínez}

Profesora Titular en el Departamento de Ciencias de la Educación de la Universidad de Alcalá (Madrid, España). Sus líneas de trabajo van encaminadas a la mejora escolar y la formación del profesorado para una educación inclusiva. En su carrera ha trabajado como maestra, jefa de estudios en la Escuela Pública y en los últimos 14 años como profesora en la Facultad de Educación formando a los futuros maestros y maestras y psicopedagogogos/as para el desarrollo de una educación Inclusiva. Ha colaborado con organismos internacionales (Agencia Europea para las necesidades educativas especiales y la educación inclusiva, OEI, etc) y en asesoramiento a distintos organismos y escuelas. Ha realizado estancias de investigación en Chile, Brasil, Reino Unido y Canadá. Cuenta con múltiples publicaciones sobre Educación Inclusiva y en la actualidad desempeña el cargo de Vicedecana $1^{\mathrm{a}}$ de la Facultad de Educación de la Universidad de Alcalá. Es miembro del Grupo de Investigación EQUIDEI. Email: yolanda.munozm@uah.es

ORCID ID: https://orcid.org/OOOO-0003-4001-02 14 